\title{
Estudo da formação de base de Schiff a partir da Quitosana com diferentes graus de desacetilação
}

Nayara Gabrielle S. Cavalcante* (Graduanda em Química na Universidade Federal do RN - UFRN); Giullia Perobelli; (Graduanda em Química na Universidade Federal do RN - UFRN);

Ana Cristina F. de Brito Pontes; (Prof. Ad. do IQ na Universidade Federal do RN - UFRN);

Daniel de Lima Pontes; (Prof. Ad. do IQ na Universidade Federal do RN - UFRN);

*E-mail: nayaragsca@gmail.com

resumo:

O trabalho caracterizou duas amostras de quitosana com diferentes graus de desacetilação. A QP possui massa molar de 4,1 x $10^{4} \mathrm{~g} / \mathrm{mol}$ e grau desacetilação de 52,06 \% enquanto a QD, mais desacetilada, possui massa molar 2,4 x $10^{4} \mathrm{~g} / \mathrm{mol}$ e 89,93 \% de desacetilação. As bases de Schiff formadas a partir da quitosana e dos aldeídos vanilina, o-vanilina e 3,4-dihidroxibenzaldeído apresentaram no infravermelho o estiramento $\mathrm{C}=\mathrm{N}$ característico de iminas em: $1634 \mathrm{~cm}^{-}$ ${ }^{1}$ (QP/OV), $1627 \mathrm{~cm}^{-1}$ (QD/OV), $1673 \mathrm{~cm}^{-1}$ (QP/vani), $1642 \mathrm{~cm}^{-1}$ (QD/vani), $1658 \mathrm{~cm}^{-1}$ (QP/DB) e $1646 \mathrm{~cm}^{-1}(\mathrm{QD} / \mathrm{DB})$.

No Uv-Vis, a QP/OV apresentou bandas referentes à ligação C=N em 308 e 339 nm. Enquanto para a QD/OV há a presença de uma banda em 213 nm que não se faz presente nessa região para a o-vanilina. Nos espectros das iminas QP/vani e QP/DB, há a inversão da intensidade de absorbância das bandas em 206 nm e 230 nm em relação às bandas dos aldeídos precursores. As bases de Schiff QD/vani e QD/DB não apresentaram no Uv-Vis diferenças significativas quando comparadas com a vanilina e o 3,4-dihidroxibenzaldeído, respectivamente.

A análise termogravimétrica revelou menor estabilidade térmica quando forma base de Schiff com a vanilina e com o 3,4-dihidroxibenzaldeído. Por outro lado, maior estabilidade térmica da quitosana quando modificada pela o-vanilina, sendo a QD/OV termicamente mais estável.

palavras-chave: Quitosana; Base de Schiff.

\section{I ntrodução}

A quitina é um dos consitituintes presentes em exoesqueletos de animais marinhos, juntamente com $\mathrm{CaCO}_{3}$, proteínas, lídeos e pigmentos ${ }^{1-3}$. É um polissacarídeo de cadeia linear, constituída quase que exclusivamente por unidades de 2-acetamino-2-desoxi-D-glicopiranose unidas por ligações $\beta$ - $(1 \rightarrow 4)^{3-4}$ (Figura 1 ). Já a quitosana é um dos principais derivados de quitina, correspondendo a um copolímero constituído de unidades 2-acetamido-2-desoxi-D-glicopiranose (GlcNAc) e 2-amino-2-desoxi-D-glicopiranose (GlcN) unidas pelo mesmo tipo de ligação glicosídica presente na quitina (Figura 1).

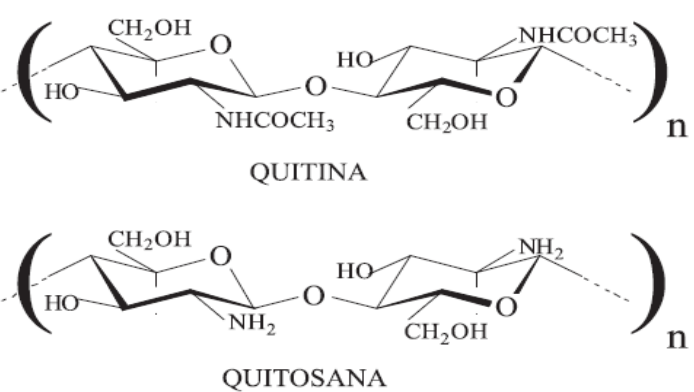

Figura 1 - Representação das unidades repetidoras de Quitina e Quitosana (Battisti, M. V.; Campana-Filho, S. P., 2008, 2014). 
A quitosana (QT) apresenta grupos funcionais potencialmente reativos: grupos amino ($\mathrm{NH}_{2}$ ), além de vários grupos hidroxilas primários e secundários nas posições C-2, C-3 e C-6, respectivamente, que apresentam forte afinidade com a água (PETER, 1995). As modificações nestes grupos produzem diferentes materiais que podem ter diversas aplicações. Dentre as modificações que a QT pode sofrer, temos, por exemplo, a formação da base de Schiff (BS). Este tipo de modificação tem chamado bastante atenção por parte dos pesquisadores, tendo em vista sua diversidade de aplicações como atividade biológica, dentre elas antibacteriana (SARI et al, 2003), antifúngica (ECHEVARRIA et al, 1999), antimicrobiana (SAKYAN et al, 2004), anti HIV (PANDEYA et al, 1999) e atividade antitumoral (DIMMOCK, 1997). As BS apresentam um papel importante na química de coordenação, pois formam complexos estáveis com vários metais de transição.

Assim, o objetivo do trabalho é realizar a modificação da quitosana purificada e desacetilada com três aldeídos: a orto-vanilina, a vanilina e o 3,4-dihidroxibenzaldeído, a fim de formar BS biopoliméricas e caracterizá-las pelas técnicas de espectroscopia na região do Infravermelho e Uv-visível, além do estudo sobre a estabilidade térmica.

\section{Metodologia}

\subsection{Método de purificação da quitosana}

O procedimento de purificação da quitosana comercial tem como base a dissolução de quitosana em ácido acético. À essa mistura, foi adicionado acetato de sódio 0,2 M. Essa suspensão foi mantida sob agitação constante por 24 horas. Após a agitação, a amostra foi filtrada em funil de placa porosa para a retirada da parte insolúvel. Etanol foi adicionado à solução filtrada, o precipitado formado foi então filtrado em funil de placa porosa, lavada com álcool etílico e acetona.

\subsection{Procedimento de desacetilação da quitosana}

O procedimento a fim de obter uma quitosana com maior grau de desacetilação seguiu, também, a metodologia de Filho e Signini (2001). Preparou-se um banho de areia com a temperatura de $110^{\circ} \mathrm{C}$ para deixar a quitosana comercial em solução de hidróxido de sódio (40 \%). Depois de 2 horas a solução foi filtrada e lavou-se o pó obtido até a água de lavagem atingir um pH neutro. A quitosana com maior grau de desacetilação também foi purificada, e para essa purificação seguiuse o mesmo método citado anteriormente.

\subsection{Determinação da Massa Molar Viscosimétrica}

Para determinações de viscosidade intrínseca, [ $\eta$ ], foi adotado o procedimento descrito por Signini e Campana Filho (2001). Utilizando a equação (Mark-Houwink) abaixo é possível a determinação da massa molar viscosimétrica.

$$
[\eta]=\mathrm{K} \cdot \mathrm{MV}_{\mathrm{v}}{ }^{\alpha}
$$

Em que, $[\eta]$ é a viscosidade intrínseca da solução, $\mathrm{K}$ é uma constante característica do polímero e depende da temperatura e do solvente, $\alpha$ é a constante característica da geometria da molécula do polímero e $\mathrm{M}_{\mathrm{v}}$ é a massa molecular viscosimétrica.

\subsection{Formação da Base de Schiff}

Bases de Schiff foram formadas pelo contato da quitosana purificada com a solução do aldeído (vanilina, orto-vanilina e 3,4-dihidroxibenzaldeído) em metanol a $50{ }^{\circ} \mathrm{C}$ por 5 horas. Em seguida, o precipitado formado foi lavado com etanol para retirar o excesso do aldeído e armazenadas em dessecador. O mesmo procedimento foi adotado para a síntese de bases de Schiff a partir da quitosana purificada com maior grau de desacetilação e os aldeídos já citados. 


\subsection{Espectroscopia na Região do I nfravermelho}

Para a obtenção de espectros na região do infravermelho foi preparada uma pastilha de $\mathrm{KBr}$ contendo cada umas das amostras do polímero, sendo elas: a quitosana purificada, quitosana purificada com maior percentual de desacetilação e as bases de Schiff provenientes duas diferentes amostras de quitosana com os aldeídos o-vanilina, vanilina, 3,4-dihidroxibenzaldeído). O equipamento utilizado para obter os espectros foi o Espectrofotômetro Shimadzu, modelo FTIR-8400S.

\subsection{Espectroscopias na Região do Ultravioleta Visível}

Os espectros na região do ultravioleta visível foram obtidos em meio aquoso utilizando o espectrofotômetro Uv-visível Agilent, modelo 8453.

\subsection{Análise Termogravimétrica e Análise Termogravimétrica Diferencial}

As analises Termogravimétrica e Termogravimétrica Diferencial foram obtidas utilizando o equipamento Analisador termogravimétrico TGA/DSC simultâneo, modelo SDT Q600.

\section{Resultados e discussão}

Filho e Signini (2001) relatam três formas distintas de purificação da quitosana: neutra, acetato e cloridrato. Em seu estudo eles caracterizaram e compararam quanto à solubilidade, hidrofilicidade, morfologia de suas superfícies e cristalinidade. A metodologia utilizada neste trabalho foi a purificação na forma de acetato por conferir a quitosana maior solubilidade. Os resultados de caracterização como grau de desacetilação (GD) e massa molar viscosimétrica $\left(\mathrm{M}_{\mathrm{v}}\right)$ da quitosana purificada (QP) e quitosana com maior grau de desacetilação (QD) são apresentados na Tabela 1.

Tabela 1 - Valores médio do grau de desacetilação (GD) e da massa molar viscosimétrica $\left(\mathrm{M}_{\mathrm{v}}\right)$ da quitosana purificada e quitosana mais desacetilada.

\begin{tabular}{c|c|c}
\hline & GD (\%) & Mv x 104 (g/mol) \\
\hline QP & 52,1 & 4,1 \\
\hline QD & 89,9 & 2,4 \\
\hline
\end{tabular}

(Fonte: próprio autor)

Figura 1 mostra a proposta da reação da QT com a vanilina, onde o grupo R é uma metoxila, o mesmo mecanismo vale para a orto-vanilina e para o 3,4-dihidroxibenzaldeído.

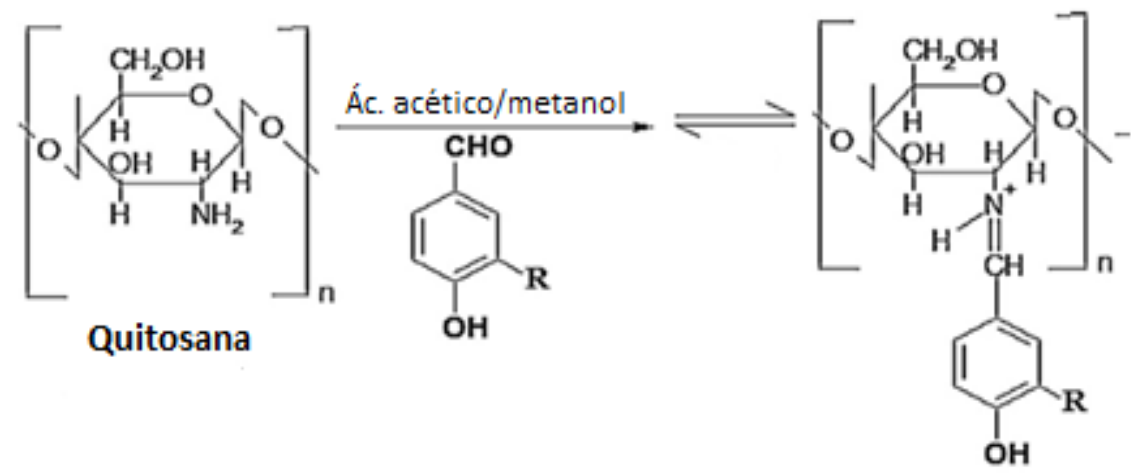

Figura 1 - Modificação química da quitosana com a vanilina (Adaptado de GUINESI, L. S., CAVALHEIRO, É. T. G., 2006, 557). 
Tabela 2 - Solubilidade dos compostos sintetizados, no qual parcialmente solúvel (PS), solúvel (S) e insolúvel (-).

\begin{tabular}{l|c|c|c|c|c|c|c}
\hline & Água & DMSO & DMF & Éter etílico & Etanol & Metanol & Ác. Acético \\
\hline QP & - & - & - & - & - & - & S \\
\hline QD & - & - & - & - & - & - & S \\
\hline QP/OV & PS & PS & - & - & - & - & S \\
\hline QD/OV & PS & PS & - & - & - & - & S \\
\hline QP/vani & PS & PS & - & - & - & - & S \\
\hline QD/vani & PS & PS & - & - & - & - & S \\
\hline QP/DB & PS & PS & - & - & - & - & S \\
\hline QD/DB & PS & PS & - & - & - & - & S \\
\hline
\end{tabular}

(Fonte: próprio autor)

A quitosana possui um caráter insolúvel em água devido a sua grande cadeia hidrocarbônica, porém também possui insolubilidade em soluções básicas e em soluções orgânicas. Por outro lado, é solúvel em soluções ácidas com pH menor que 6 devido a protonação do grupo amino $\left(\mathrm{NH}_{2}\right)$, gerando uma espécie catiônica e mais solúvel em solventes polares, sendo comumente utilizado o ácido acético para solubilização desse polímero. Após as modificações feitas no grupo amino da QT a fim de formar o grupo imina $(\mathrm{C}=\mathrm{N})$, observou-se que as bases de Schiff seguem o mesmo padrão de solubilidade apresentado pela quitosana, com a exceção para a parcial solubilidade em dimetilsulfóxido (DMSO) e em água.

O estudo da caracterização da modificação química da QT foi realizado por espectroscopia vibracional na região do infravermelho (IV) e espectroscopia eletrônica na região do ultravioleta e visível (Uv-Vis). A Figura 2 mostra o espectro de infravermelho para as amostras de quitosana (2a) purificada e desacetilada, já a figura 2b mostra os espectros para as amostras de quitosana modificadas.

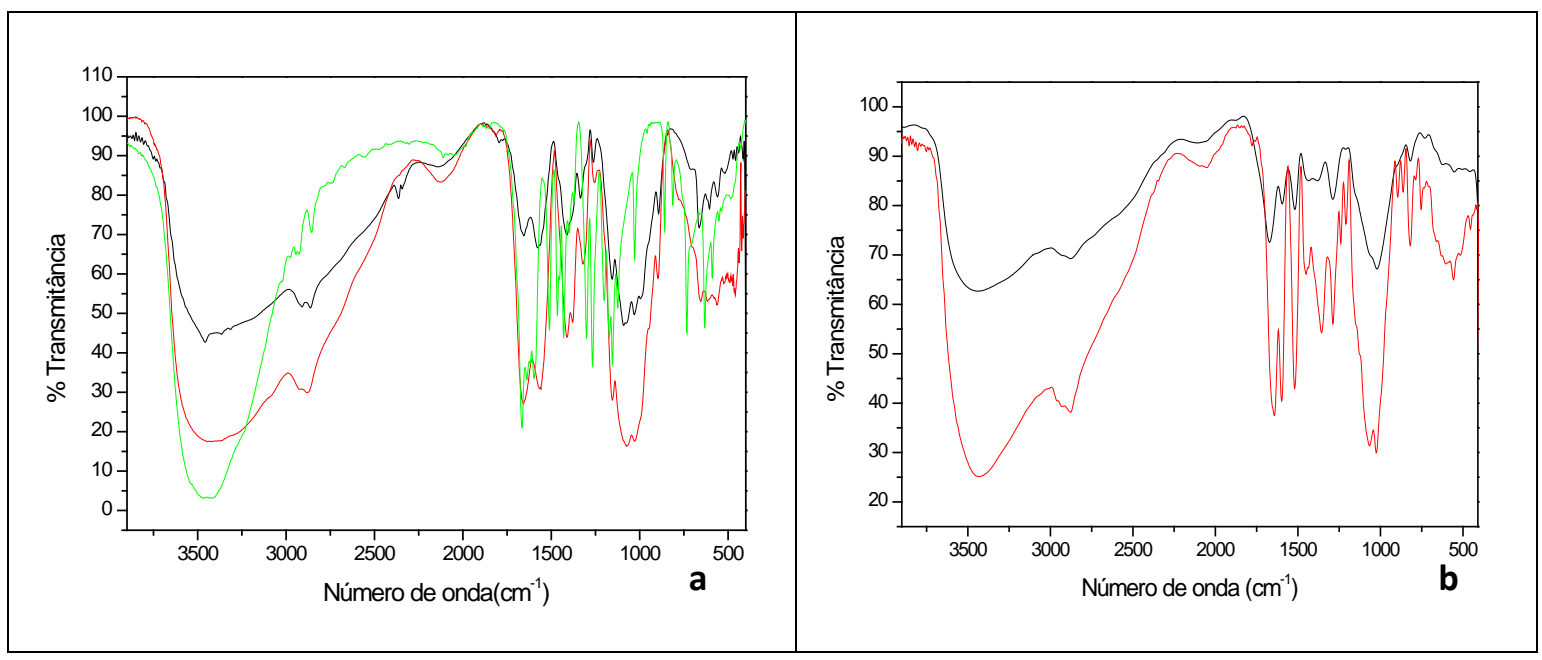

Figura 2 - Espectro de Infravermelho em pastilha de $\mathrm{KBr}$ : (a) quitosana purificada (-), quitosana desacetilada purificada (-) e vanilina (-); (b) Bases de Schiff da QP(-), QD (b) (-).

Tabela 3 - Atribuições das bandas de infravermelho para as amostras de quitosana purificadas e dos aldeídos: vanilina, o-vanilina e 3,4-dihidroxibenzaldeído ( $\delta=$ deformação; $v=$ estiramento). 


\section{ATRIBUIÇÕES DAS BANDAS IV $\left(\mathrm{cm}^{-1}\right)$}

\begin{tabular}{|c|c|c|c|c|c|c|c|}
\hline Quitosana & $\begin{array}{l}V_{\mathrm{C}=\mathbf{O}} \\
\text { (amida I) }\end{array}$ & $\begin{array}{c}\delta_{\mathrm{N}-\mathrm{H}} \\
\text { (amida II) }\end{array}$ & $\begin{array}{c}\delta_{\mathrm{C}-\mathrm{N}} \\
\text { (amida III) }\end{array}$ & $V_{\operatorname{COC} \beta-(1-4)}$ & $\delta_{\mathrm{CO}}$ & $v_{\text {OH }}$ & $v_{C-H}$ \\
\hline$\overline{Q P}$ & 1654 & 1553 & 1407 & 1155 & 1064 & 3420 & 2871 \\
\hline QD & 1653 & 1573 & 1412 & 1150 & 1090 & 3456 & 2857 \\
\hline Bases de Schiff & $\begin{array}{c}\mathrm{V}_{\mathrm{C}=\mathrm{O}} \\
\text { (aldeído) }\end{array}$ & $\begin{array}{l}\delta_{\mathrm{N}-\mathrm{H}} \\
\text { (amina) }\end{array}$ & $\begin{array}{c}\delta_{\mathrm{C}-\mathrm{N}} \\
\text { (imina) }\end{array}$ & $V_{\operatorname{COC} \beta-(1-4)}$ & $\delta_{\mathrm{co}}$ & $v_{\mathrm{OH}}$ & $v_{\text {C-H }}$ \\
\hline Vanilina & 1661 & - & - & - & 1161 & 3440 & 2856 \\
\hline O-vanilina & 1639 & - & - & - & 1069 & 3014 & 2971 \\
\hline DB & 1654 & - & - & - & 1156 & 3285 & 2868 \\
\hline
\end{tabular}

(Fonte: próprio autor)

A tabela 3 apresenta as principais bandas características da QP, QD e dos aldeídos. Podese observar a sobreposição de muitas bandas entre os aldeídos e a quitosana. Os deslocamentos observados são condizentes com os valores relatados na literatura.

Os outros dois aldeídos utilizados para a modificação química foram a orto-vanilina e o 3,4 - dihidroxibenzaldeido, a Figura 3 mostra os IV obtidos para as bases de Schiff obtidas para a QP e QD.

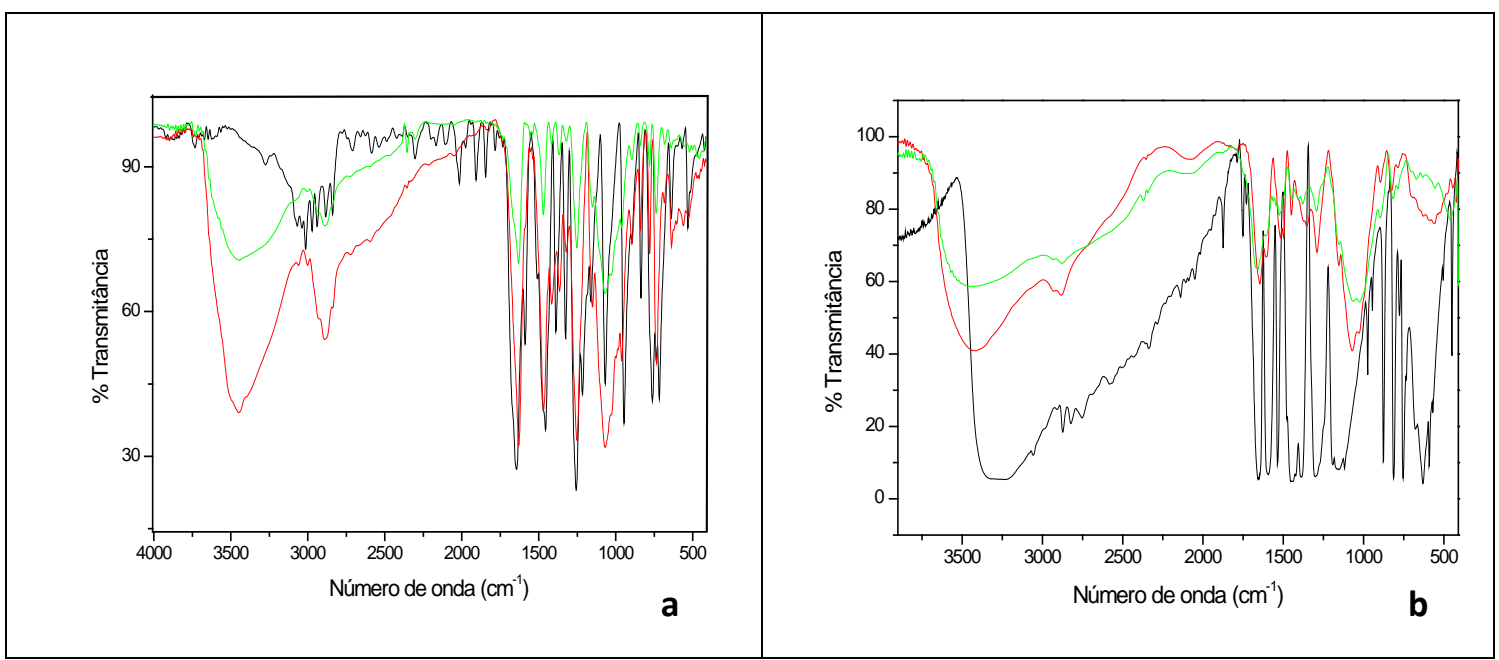

Figura 3 - Espectro de Infravermelho em pastilha de KBr: (a) para orto-vanilina (-), BS QP/OV (-) e BS QD/OV (-); (b) para 3,4-dihidroxibenzaldeido (-), BS QP/DB (-) e BS QD/DB (-).

As principais bandas que são observadas no espectro de IV da BS QP/vani: estiramento $\mathrm{O}-\mathrm{H}$ resultante de associação polimérica $\left(3444 \mathrm{~cm}^{-1}\right)$, estiramento $\mathrm{C}=\mathrm{N}\left(1673 \mathrm{~cm}^{-1}\right)$, o estiramento C-O de álcool secundário da quitosana $\left(1021 \mathrm{~cm}^{-1}\right)$, o estiramento C-O de éter aromático da vanilina $\left(1290 \mathrm{~cm}^{-1}\right)$.

Por outro lado, a QD/vani possui deslocamentos em suas bandas em relação à QP/vani, apresentando o estiramento O-H resultante de associação polimérica em $3436 \mathrm{~cm}^{-1}$, estiramento 
$\mathrm{C}=\mathrm{N}$ em $1642 \mathrm{~cm}^{-1}$, estiramento C-O de álcool secundário da quitosana em $1024 \mathrm{~cm}^{-1}$ e o estiramento C-O de éter aromático da vanilina em $1288 \mathrm{~cm}^{-1}$.

As principais bandas identificadas na BS QP/OV (Figura 3a) são: estiramento O-H resultante de associação polimérica $\left(3444 \mathrm{~cm}^{-1}\right)$, estiramento $\mathrm{C}=\mathrm{N}\left(1634 \mathrm{~cm}^{-1}\right)$, além do estiramento C-O de álcool secundário da QT $\left(1069 \mathrm{~cm}^{-1}\right)$, o estiramento C-O de éter aromático da OV $\left(1249 \mathrm{~cm}^{-1}\right)$ e banda referente à substituição orto no anel aromático $\left(737 \mathrm{~cm}^{-1}\right)$.

Enquanto na QD/OV as principais bandas identificadas são: estiramento O-H resultante de associação polimérica $\left(3449 \mathrm{~cm}^{-1}\right)$, estiramento $\mathrm{C}=\mathrm{N}\left(1627 \mathrm{~cm}^{-1}\right)$, o estiramento C-O de álcool secundário da quitosana $\left(1070 \mathrm{~cm}^{-1}\right)$, o estiramento C-O de éter aromático da orto-vanilina (1249 $\left.\mathrm{cm}^{-1}\right)$ e banda referente à substituição orto no anel aromático $\left(735 \mathrm{~cm}^{-1}\right)$.

Já a BS QP/DB possui como principais bandas no IV estiramento O-H resultante de associação polimérica $\left(3444 \mathrm{~cm}^{-1}\right)$, estiramento $\mathrm{C}=\mathrm{N}\left(1658 \mathrm{~cm}^{-1}\right)$, o estiramento C-O de álcool secundário da quitosana $\left(1062 \mathrm{~cm}^{-1}\right)$, o estiramento C-O de éter aromático do 3,4dihidroxibenzaldeído $\left(1293 \mathrm{~cm}^{-1}\right)$.

Para a base de Schiff obtida da quitosana desacetilada, QD/DB, as principais bandas no IV são: estiramento O-H resultante de associação polimérica $\left(3419 \mathrm{~cm}^{-1}\right)$, estiramento $\mathrm{C}=\mathrm{N}(1646$ $\left.\mathrm{cm}^{-1}\right)$, o estiramento C-O de álcool secundário da quitosana $\left(1070 \mathrm{~cm}^{-1}\right)$, o estiramento C-O de éter aromático do 3,4-dihidroxibenzaldeído $\left(1288 \mathrm{~cm}^{-1}\right)$.

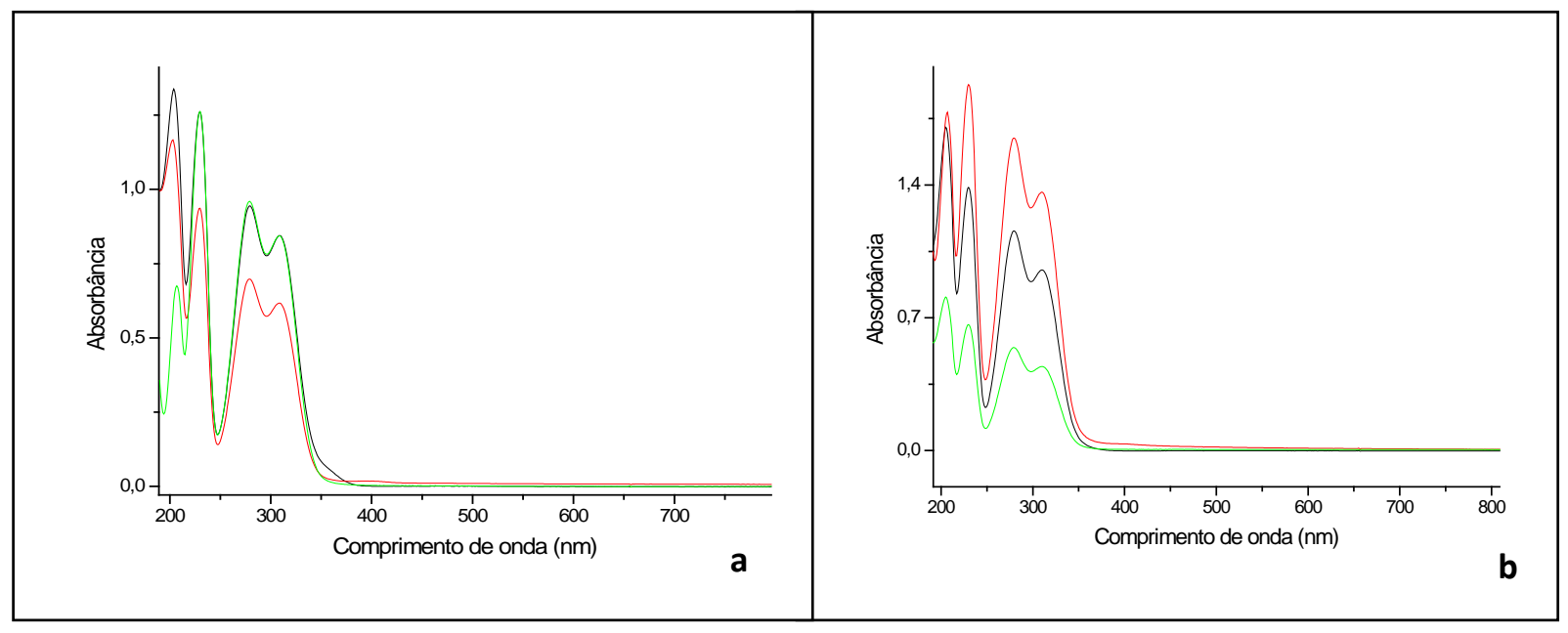

Figura 4 - Espectro de Uv-Vis na região de 200 à $800 \mathrm{~nm}$ em meio aquoso: (a) para vanilina (-), BS QP/vani (-) e BS QD/vani (-); (b) para 3,4-dihidroxibenzaldeido (-), BS QD/DB (-) e BS QP/DB (-).

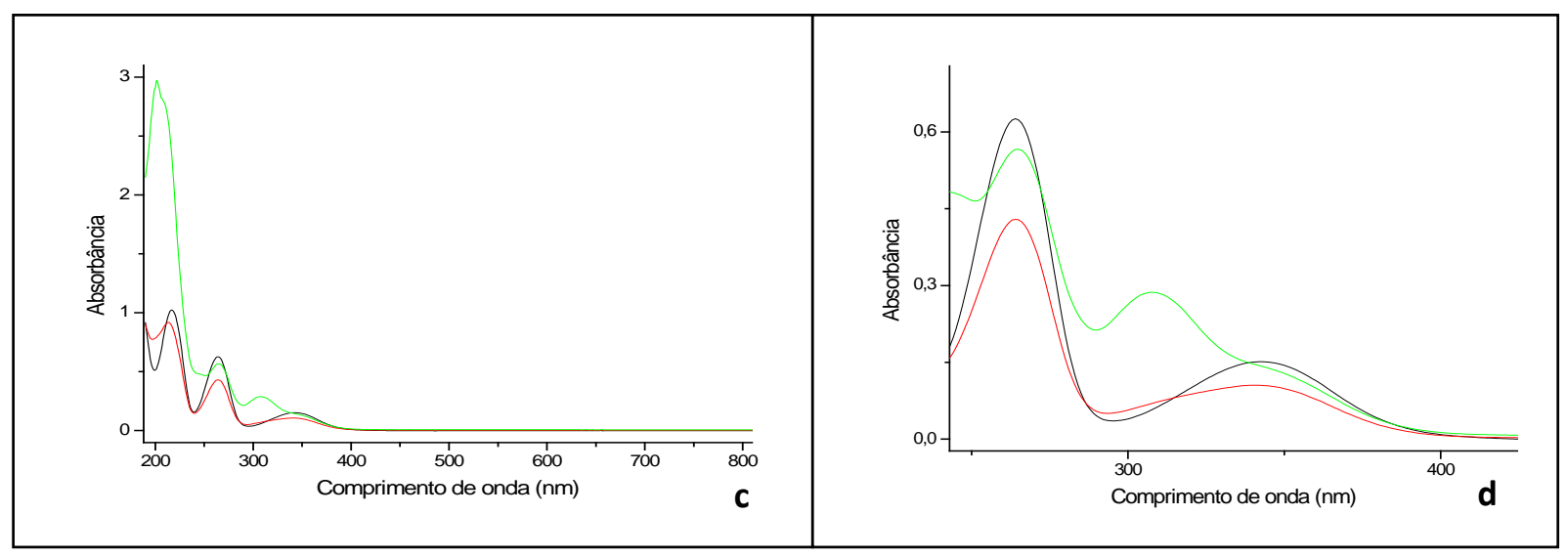


Figura 5 - Espectro de Uv-Vis na região de 200 à $800 \mathrm{~nm}$ em meio aquoso: (c) para o-vanilina (-), BS QP/OV (-) e BS QD/OV (-); (d) ampliação do espectro (c) na região de 250 à 400 nm.

No Uv-Vis, as bases de Schiff QP/vani e QD/vani apresentaram um espectro semelhante ao do aldeído vanilina com bandas em 206 nm, 230 nm, 279 nm e 310 nm. Porém as bandas em 206 nm e 230 nm da QP/vani possuem inversão na intensidade de absorbância em relação às bandas presentes nessa região para a vanilina.

Enquanto para a QP/OV, houve o aparecimento de quatro bandas (205, 265, 308 e 339 $\mathrm{nm}$ ), sendo duas bandas referentes ao grupo funcional $\mathrm{C}=\mathrm{N}$ em 308 e $339 \mathrm{~nm}$, enquanto a banda referente ao grupo $\mathrm{C}=\mathrm{O}$ da $\mathrm{OV}$ (342 nm) não se faz presente. Já o espectro da QD/OV, apresenta bandas muito semelhantes as da orto-vanilina (217, 264 e 342 nm). Porém, em seu espectro há a presença de uma banda em 213 nm e ausência em 217 nm.

Por outro lado, a modificação da quitosana com o 3,4-dihidroxibenzaldeído, assim como a modificação com a vanilina, apresentou compostos com espectros semelhantes ao do aldeído precursor, porém, para a BS QP/DB há inversão de intensidade de absorbância das bandas em 205 e 230 nm em relação às bandas presentes nessa região para o aldeído.

As amostras de quitosana e as modificações com os aldeídos foram submetidos à análises termogravimétricas.

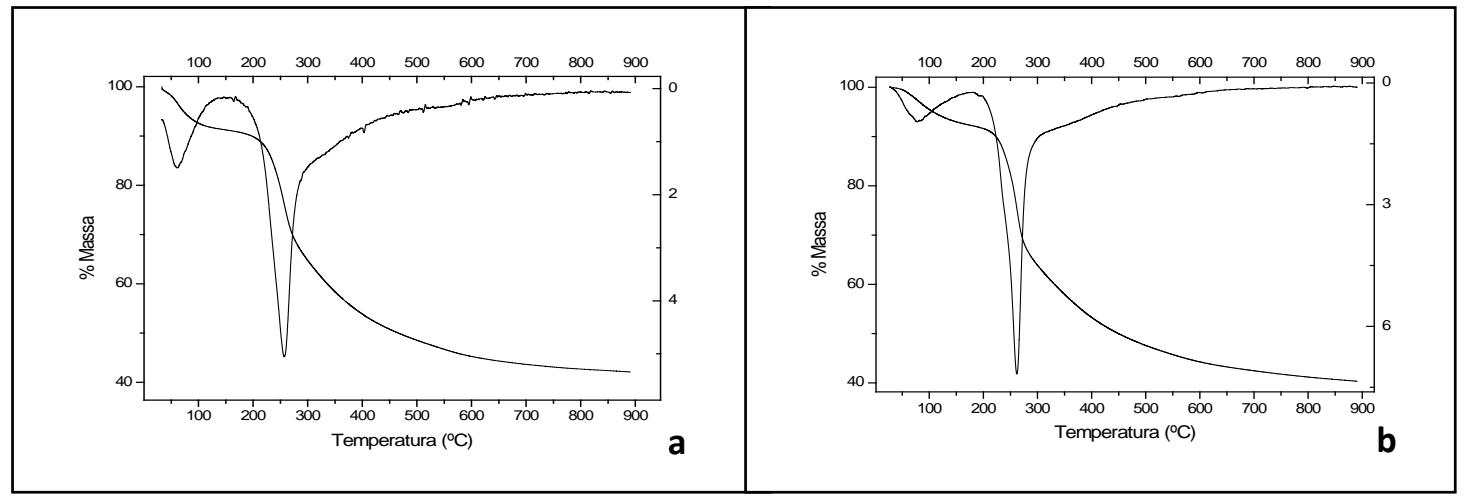

Figura 6 - Análise termogravimétrica e análise termogravimétrica diferencial: (a) BS QP/vani; (b) BS QD/vani.

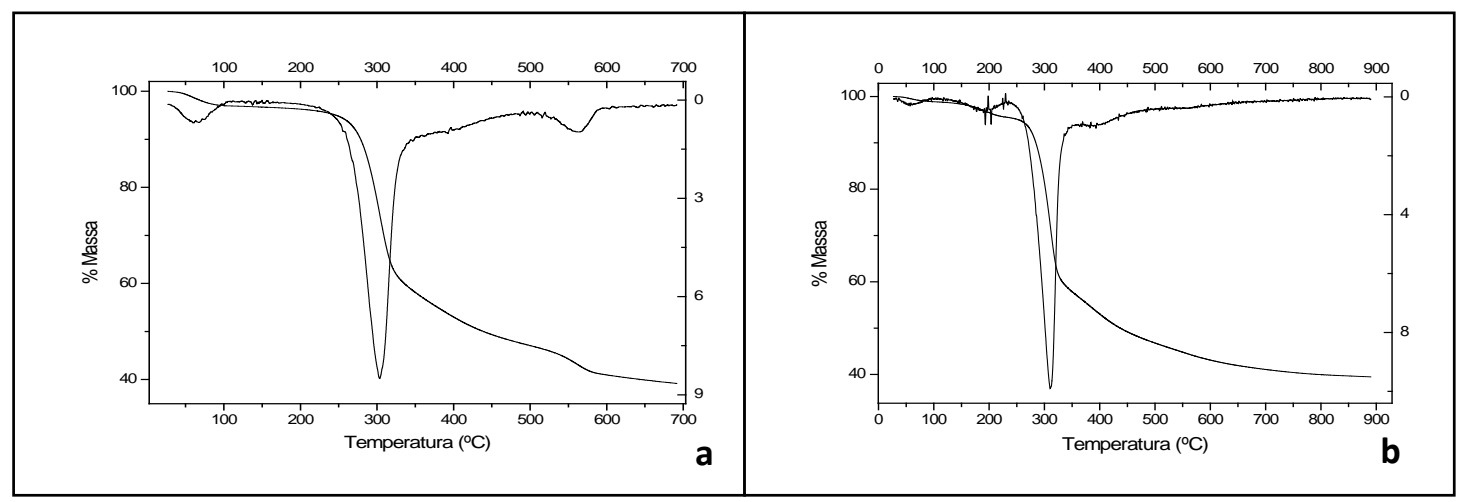

Figura 7 - Análise termogravimétrica e análise termogravimétrica diferencial: (a) BS QP/OV; (b) BS QD/OV. 


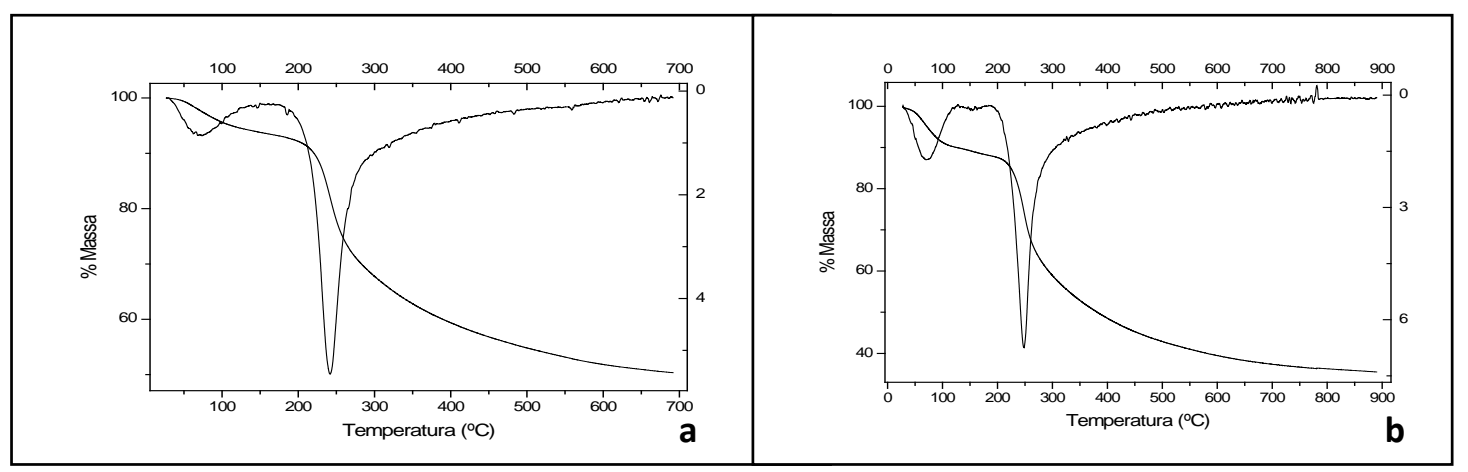

Figura 8 - Análise termogravimétrica e análise termogravimétrica diferencial: (a) BS QP/DB; (b) BS QD/DB.

Tabela 4 - Valores atribuídos às temperaturas de decomposição das amostras de quitosana e das modificações com os aldeídos.

\begin{tabular}{c|c|c|c|c|c|c|c|c}
\hline & $\mathrm{QP}$ & $\mathrm{QP} / \mathrm{vani}$ & $\mathrm{QP} / \mathrm{OV}$ & $\mathrm{QP} / \mathrm{DB}$ & $\mathrm{QD}$ & $\mathrm{QD} / \mathrm{vani}$ & $\mathrm{QD} / \mathrm{OV}$ & QD/DB \\
\hline Temperatura $\left({ }^{\circ} \mathrm{C}\right)$ & 255 & 257 & 304 & 242 & 288 & 262 & 311 & 248 \\
\hline
\end{tabular}

(Fonte: próprio autor)

A análise termogravimétrica (TG) indica a saída de água adsorvida sobre as amostras próximo a $100{ }^{\circ} \mathrm{C}$. Além dessa perda de massa, observa-se também as perdas significativas de massa devido às degradações dos compostos com o aumento da temperatura. Assim, com base nos dados de temperatura para os eventos de degradação de cada composto, pode-se afirmar que as bases de Schiff formadas com os aldeídos vanilina e 3,4-dihidroxibenzaldeído possuem menor estabilidade frente à temperatura em relação às duas formas de quitosana antes da reação. Diferentemente do que acontece com as iminas formadas a partir da orto-vanilina, pois ambas apresentam maior estabilidade térmica, sendo a imina QD/OV a que possui maior estabilidade.

\section{Conclusão}

Por meio do método de viscosidade intrínseca e titulação condutométrica obteve-se, respectivamente, a massa molar, 4,1 x $10^{4} \mathrm{~g} / \mathrm{mol}$, e o grau de desacetilação, 52,06 \%, da quitosana comercial purificada. Após a realização do processo de desacetilação da QT purificada, objetivando um maior número de grupamentos amino livres, obteve-se uma quitosana com massa molar igual a $2,4 \times 10^{4} \mathrm{~g} / \mathrm{mol}$ e grau de desacetilação de 89,93 \%.

A fim de formar bases de Schiff, as duas amostras de quitosana (QP e QD) reagiram com os aldeídos: vanilina, o-vanilina e o 3,4-dihidroxibenzaldeído. As modificações são indicadas pela presença do estiramento $\mathrm{C}=\mathrm{N}$ em $1634 \mathrm{~cm}^{-1}(\mathrm{QP} / \mathrm{OV}), 1627 \mathrm{~cm}^{-1}(\mathrm{QD} / \mathrm{OV}), 1673 \mathrm{~cm}^{-1}$ (QP/vani), $1642 \mathrm{~cm}^{-1}$ (QD/vani), $1658 \mathrm{~cm}^{-1}(\mathrm{QP} / \mathrm{DB})$ e $1646 \mathrm{~cm}^{-1}$ (QD/DB), que não é observado na quitosana.

Por outro lado, o Uv-Vis indica a formação das iminas QP/OV, QD/OV, QP/vani e QP/DB por meio de modificações visualizadas a partir da comparação entre os espectros dos aldeídos precursores e as bases de Schiff, indicando mudança no ambiente químico. Enquanto a iminas QD/vani e QD/DB apresentam espectros semelhantes ao do aldeído precursor. A análise termogravimétrica dos compostos revelou que a modificação da quitosana com a orto-vanilina aumentou sua estabilidade térmica, mas por outro lado, diminui quando modificada com os outros aldeídos. 


\section{Referências bibliográficas}

SANTOS, J. E.; SOARES, J. P.; DOCKAL, E. R.; CAVALHEIRO, E. T. G.; FILHO, S. P. C. Caracterização de Quitosanas Comerciais de Diferentes Origens, Polímeros: Ciência e Tecnologia, 13, 4, 2003.

PETER, M. G. Applications and environmental aspects of chitin and chitosan, J. Macromol SciPure e Appl. Chem., 32, 4, 1995.

ECHEVARRIA, A.; NASCIMENTO, M. G.; GERÔNIMO, V.; Miller, J. e GIESBRECHT, A. NMR Spectroscopy, Hammett Correlations and Biological Activity of Some Schiff Bases Derived from Piperonal, J. Braz. Chem. Soc., 10, 1, 1999.

SAKYAN, I.; LOGOGLU, E.; ARSLAN, S.; SARI, N. e SAKIYAN, N. Antimicrobial activities of N(2-hydroxy-1-naphthalidene)-amino acid(glycine, alanine, phenylalanine, histidine, tryptophane) Schiff bases and their manganese(III) complexes, Biometals, 17, 2, 2004.

PANDEYA, S. N.; SRIRAM, D.; NATH, G.; CLERCQ, E. Synthesis, antibacterial, antifungal and antiHIV evaluation of Schiff and Mannich bases of isatin derivatives with 3-amino-2-methylmercapto quinazolin-4(3H)-one, Pharmaceutica Acta Helvetiae, 74, 1, 1999.

DIMMOCK, J. R.; KUMAR, P. Anticancer and Cytotoxic Properties of Mannich Bases, Curr. Med. Chem, 4, 1, 1997.

SI GNI NI, R.; CAMPANA-FILHO, S. P. Características e Propriedades de Quitosanas Purificadas nas Formas Neutra, Acetato e Cloridrato, Polímeros: Ciência e Tecnologia, 11, 2, 2001.

BATTISTI, M. V.; CAMPANA-FILHO, S. P. Obtenção e caracterização de a-quitina e quitosanas de cascas de Macrobrachium rosembergii. Química Nova, 31, 8, 2008.

GUI NESI, L. S., CAVALHEIRO, É. T. G. Influence of some reactional parameters on the substitution degree of biopolymeric Schiff bases prepared from chitosan and salicylaldehyde, Carbohydrate Polymers, 65, 4, 2006.

MATHUR, N. K.; NARANG, C. K. Chitin and chitosan, versatile polysaccharides from marine animals, J. Chem. Educ., 67, 11, 1990.

PERCOT, A.; VITON, C.; DOMARD, A. Optimization of Chitin Extraction from Shrimp Shells, Biomacromolecules, 4, 1, 2003.

EINBU, A.; NAESS, S. N.; ELGSAETER, A.; VARUM, K. M. Solution Properties of Chitin in Alkali, Biomacromolecules, 5, 5, 2004.

ZHANG, M.; HAGA, A.; SEKIGUCH, H.; HIRANO, S. Structure of insect chitin isolated from beetle larva cuticle and silkworm (Bombyx morl) pupa exuvia, Int. J. Biol. Macromol, 27, 1, 2000.

DAMIAN, C.; BEIRÃO, L. H.; FRANCISCO, A. SANTO, M. L. P. E.; TEIXEIRA, E. Quitosana: um amino polissacarídio com características funcionais, Alim. Nutr., 16, 2, 2005.

\section{Study of Schiff base formation from Chitosan of different degrees of deacetylation}

\section{Abstract:}

The work featured two samples of chitosan with different degrees of deacetylation. QP has molar mass of $4.1 \mathrm{x} 104 \mathrm{~g} / \mathrm{mol}$ and deacetylation degree of $52.06 \%$ while the QD plus deacetylated, has a molar mass of $2.4 \times 104 \mathrm{~g} / \mathrm{mol}$ and deacetylation of $89.93 \%$. The Schiff bases formed 
from aldehydes of chitosan and vanillin, o-vanillin, and 3,4- dihidroxibenzaldeído infrared showed the characteristic $\mathrm{C}=\mathrm{N}$ stretch in imines: $1634 \mathrm{~cm}^{-1}(\mathrm{QP} / \mathrm{OV}), 1627 \mathrm{~cm}^{-1}$ (QD/OV), 1673 $\mathrm{cm}^{-1}$ (QP/vani), $1642 \mathrm{~cm}^{-1}$ (QD/vani), $1658 \mathrm{~cm}^{-1}$ (QP/DB) e $1646 \mathrm{~cm}^{-1}$ (QD/DB).

$\mathrm{UV}-\mathrm{Vis} \mathrm{QP} / \mathrm{OV}$ showed bands related to the $\mathrm{C}=\mathrm{N}$ bond at 308 and $339 \mathrm{~nm}$. As for the $\mathrm{QD} / \mathrm{OV}$ there is the presence of a band at $213 \mathrm{~nm}$ which is not present in this region for o- vanillin. In the spectra of imines $\mathrm{QP} / \mathrm{vani}$ and $\mathrm{QP} / \mathrm{DB}$, there is the inversion of the intensity of absorbance bands at $206 \mathrm{~nm}$ and $230 \mathrm{~nm}$ over the bands of the precursor aldehydes. Schiff bases QD/vani and QD/DB does not show the UV- Vis significant differences compared to vanillin and 3,4dihidroxibenzaldeído respectively.

Thermogravimetric analysis revealed a lower thermal stability when forming Schiff base and vanillin with 3,4- dihidroxibenzaldeído . On the other hand, greater thermal stability when modified chitosan o-vanillin, and QD/OV more thermally stable.

Keywords: Chitosan; Schiff base. 